\title{
Shift of the Shadow Boundary in High Frequency Scattering
}

\section{Maciej Zworski}

Department of Mathematics, Harvard University, Cambridge, MA 02138, USA

Received March 13, 1990; in revised form April 11, 1990

Abstract. The microlocal theory of diffraction is used to establish the conjecture of Keller and Rubinow relating the shift of the shadow boundary in high frequency scattering to the directional curvatures of a strictly convex obstacle.

\section{Introduction and Statement of Results}

In their paper on scattering by a cylinder [3], Keller and Rubinow conjecture that the shift of the shadow cast by any object is asymptotically equal to

$$
C \alpha^{-1 / 3} \lambda^{-2 / 3}
$$

along each glancing ray, where $\lambda$ is the frequency and $\alpha$ is the directional curvature of the object at the glancing point at the direction of the glancing ray. The constant $C$ depends only on the boundary condition at the glancing point and is positive for the Dirichlet problem (hard obstacle), negative for the Neumann problem (soft obstacle), i.e. the shadow boundary is shifted outwards or inwards respectively.

The work of Keller and Rubinow followed earlier computations of Artmann, Rice and Logan. For scattering by a sphere in $\mathbf{R}^{3}$, the conjecture was proved by Nussenzveig [10].

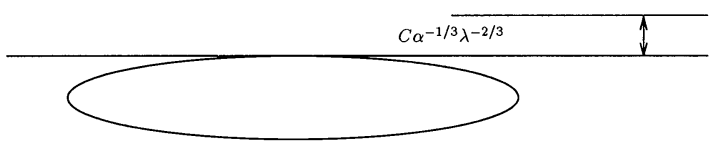

Fig. 1

The purpose of this note is to establish the asymptotic shift of the shadow boundary for scattering by any strictly convex obstacle in $\mathbf{R}^{n}$. This is done using 
the microlocal theory of diffraction developed by Melrose and Taylor [7-9]. The analogous conjecture of Keller and Rubinow for the scattering amplitude was established by Melrose [5]. Our analysis is also related to the study of uniform expansions of the stationary wave diffracted by a convex obstacle $[8,11]$, although, rather fortunately, we can find simpler expansions sufficient for the investigation of the size of the diffracted wave.

Let $w_{F}(x, \lambda)$ be the free plane wave in the direction $\omega$, and let $w(x, \lambda)$ be the corresponding diffracted stationary wave. (Precise definitions are presented in Sect. 2.) At infinite frequency the amplitude of the diffracted wave, $|w(x, \lambda)|$, on the shadow boundary is equal to half the amplitude of the free wave. This suggests a definition [3] of the "physical" shadow boundary at finite frequencies as the locus of points $x$ (near the shadow) where

$$
|w(x, \lambda)|=\frac{1}{2}\left|w_{F}(x, \lambda)\right| \text {. }
$$

Let $\bar{s}(x)$ denote the (Euclidean) distance to the shadow boundary, defined so that it is negative in the shadow (see Sect. 3). As will be shown in Sect.4, for $x$ on the shadow boundary, $\bar{s}(x)=0$, and away from the obstacle,

$$
|w(x, \lambda)|=\left(\frac{1}{2}+O\left(\lambda^{-1 / 6}\right)\right)\left|w_{F}(x, \lambda)\right|+O\left(\lambda^{-1}\right) .
$$

The theorem below provides the asymptotic correction term in agreement with the conjecture of Keller and Rubinow:

Theorem 1. For $x$ away from the obstacle, $d(x, \partial K)>\delta$, and

$$
\bar{s}(x)=C\left(\varrho(y(x))[\alpha(y(x), \omega)]^{-1 / 3} \lambda^{-2 / 3},\right.
$$

we have

$$
|w(x, \lambda)|=\left(\frac{1}{2}+O\left(\lambda^{-1 / 3}\right)\right)\left|w_{F}(x, \lambda)\right|+O\left(\lambda^{-1}\right) .
$$

Here, $\omega$ is the direction of the plane wave, $y(x)$ is the point on $\partial K$ at which the ray on the shadow boundary closest to $x$ meets $\partial K$, and $\alpha(y, \omega)$ is the directional curvature of $\partial K$ at $y$ in the direction $\omega$. The constant $C$ depends only on the boundary condition at $y(x)$ :

$$
\left.\left(\varrho u+\partial_{v} u\right)\right|_{\partial K}=0,
$$

and, with $\varrho=\infty$ corresponding to the Dirichlet boundary condition ${ }^{1}$ :

$$
C(0)<0 \quad C(\infty)>0 \text {. }
$$

In the case most interesting in scattering theory, and the one considered by Keller and Rubinow, $w_{F}(x, \lambda)=\exp (i x \cdot \omega)$ so that we obtain

$$
|w(x, \lambda)|=\frac{1}{2}+O\left(\lambda^{-1 / 3}\right) \text {. }
$$

We conclude this section with several remarks. One could consider the surface giving the "physical" shadow boundary:

$$
\Gamma_{\lambda}=\left\{x:|w(x, \lambda)|=\frac{1}{2}\left|w_{F}(x, \lambda)\right|\right\},
$$

1 The numerical values of these constants have been computed (see [3] for references) and $C(0) \approx$ -1.180589 and $C(\infty) \approx 1.360825$ 
and the distance function to $\Gamma_{\lambda}, \bar{s}_{\lambda}(x)$. Then Theorem 1 provides the first term in the expansion of $\bar{s}_{\lambda}(x)$ :

$$
\bar{s}_{\lambda}(x)=\bar{s}(x)-C\left(\varrho ( y ( x ) ) \left[\alpha(y(x), \omega]^{-1 / 3} \lambda^{-2 / 3}+O\left(\lambda^{-5 / 6}\right) .\right.\right.
$$

If instead of a plane wave one took a more general initial Lagrangian satisfying appropriate geometric conditions (see $[8,11])$ then one could generalize Theorem 1 appropriately. The shift would then depend on $x$ but the only dependence on the obstacle would still come from the multiple of the cubic root of the directional curvature. Similar generalization is possible in the use of varying the metric $g$ in $\mathbf{R}^{n}$ and the Laplacian $\Delta_{g}$.

We should also remark that using the methods of [11] (in particular Proposition 7.2 and Theorem 8.1 in Propositions 4 and 5 below, respectively) we could weaken the assumption $d(x, \partial K)>\delta$ to $d(x, \partial K)>\lambda^{-1 / 3}$ at the expense of changing the error term in Theorem 1 to $O\left(\left(\lambda^{1 / 3} d(x, \partial K)\right)^{-1}\right)$. Unlike the methods presented below this requires a rather technical approach and does not seem physically significant.

\section{Preliminaries}

In this section we shall specify the assumptions on the solution to the boundary problem and recall an expression for that solution. We shall be concerned with the following situation. Let $K$ be a smooth, compact and strictly convex set in $\mathbf{R}^{n}$, and let us consider the mixed problem in the exterior domain $\mathcal{O}=\mathbf{R}^{n} \backslash K$ :

$$
\begin{gathered}
\left(D_{t}^{2}-\Delta\right) u=0 \quad \text { in } \quad \mathbf{R}_{+} \times \mathcal{O}, \\
\left.\left(\varrho+\partial_{v} u\right)\right|_{\mathbf{R}_{+} \times \partial \mathcal{O}}=0, \\
\left.u\right|_{t=0}=f, \\
\left.D_{t} u\right|_{t=0}=g,
\end{gathered}
$$

where $\varrho \in C^{\infty}(\partial K)$ with $\varrho=\infty$ corresponding to the Dirichlet problem and where $f, g$ are compactly supported in $\mathcal{O}$ and

$$
f \in I_{\mathrm{cl}}^{\alpha}(\mathcal{O}, \Lambda), \quad g \in I_{\mathrm{cl}}^{\alpha+1}(\mathcal{O}, \Lambda), \quad \Lambda=N^{*}\{x \cdot \omega+a=0\},
$$

where $I_{\mathrm{cl}}^{m}(\mathcal{O}, \Lambda)$ denotes the space of classical Lagrangian distributions associated to the conic Lagrangian $\Lambda \subset T^{*} \mathcal{O}$. The free nonhomogeneous and homogeneous flow-outs of $\Lambda$ are respectively:

$$
\begin{aligned}
& \Lambda_{D}=\left\{(x, \omega): x \in \mathbf{R}^{n}\right\} \subset T^{*}\left(\mathbf{R}^{n}\right), \\
& \tilde{\Lambda}_{D}=\left\{(x, x \cdot \omega+a ; \lambda \omega, \omega): x \in \mathbf{R}^{n}\right\} \subset T^{*}\left(\mathbf{R}^{n+1}\right) .
\end{aligned}
$$

We can now define the geometric shadow, identifying $\Lambda_{D}$ with the free rays, $M^{0}=\{x+t \omega: t \in \mathbf{R}\}$,

$$
\Gamma=\pi\left(\left\{p \in M^{0}: \text { the ray } p \text { is tangent to } \partial K\right\}\right),
$$

where as before $\pi: s^{*} \mathbf{R}^{n} \rightarrow \mathbf{R}^{n}$, and by our assumptions on $\Lambda, \Gamma$ is a hypersurface. We also define

$$
\bar{s}(x)=d(x, \Gamma),
$$

with $d$ denoting the (Euclidean) distance. 
The stationary wave is the Fourier transform of the solution of the wave equation:

$$
w(x, \lambda)=\int_{0}^{\infty} u(t, x) e^{-i t \lambda} d t,
$$

and as in [8] for $x$ in a compact set the standard results on propagation of singularities show that one only needs to consider the above integral with a compactly supported cut-off function in $t$ inserted.

The usual scattering problem and the one considered by Keller and Rubinow corresponds to

$$
\begin{aligned}
& f=\delta(x \cdot \omega+a), \\
& g=i \delta^{\prime}(x \cdot \omega+a),
\end{aligned}
$$

and $x \cdot \omega+a>0$ on $\partial K$. This could be rephrased as

$$
u(t, x)=\delta(x \cdot \omega+a-t) \text { for } t \ll 0 .
$$

The free wave is the solution of the problem

$$
\begin{gathered}
\left(D_{t}^{2}-\Delta\right) u_{F}=0 \text { in } \mathbf{R}_{+} \times \mathcal{O}, \\
\left.u_{F}\right|_{t=0}=f \\
\left.D_{t} u_{F}\right|_{t=0}=g
\end{gathered}
$$

and similarly

$$
w_{F}(x, \lambda)=\int_{0}^{\infty} u_{F}(t, x) E^{-i t \lambda} d t .
$$

The Melrose-Taylor parametrix for the diffractive boundary problems provides the microlocal description of the wave group, i.e. the solution $u(t, x)$ above - see $[1,8,11]$. As in the case of the Dirichlet parametrix that requires a special solution of the eikonal equation (see Sect. 3). Here, particularly important is the expression of the free solution using the same phase function (see Lemma 3.1 and formula (7.2) in [11]).

Since the main object for us is the relation between the symplectic construction (the phase function) and the geometry of the scatterer (the directional curvature) we shall review the construction of the phase function in Sect.3. However we shall only quote [8] (formula (3.8)) and [11] (Proposition 4.2 and formula (7.2)) for the expression of $w$ and $w_{F}$ away from $\partial K$ and close to the geometric shadow boundary, $\Gamma_{+}$. For simplicity we shall restrict ourselves to the cases $\varrho=0$ and $\varrho=\infty$ (see Sect. 4 for comments on the general boundary condition).

Proposition 1. For $d(x, \partial K)>\delta$ and $x$ close to $\Gamma_{+}$we can find $g \in S_{p g h}^{0}\left(\mathcal{O}_{x} \times \mathbf{R}_{\eta} ; \mathbf{R}_{\lambda}\right)$, compactly supported in $\eta$ such that for $\varrho=\infty$,

$$
\begin{aligned}
w(x, \lambda) & \equiv-\omega^{2} \lambda^{\alpha+n / 4} \int g(x, \eta ; \lambda) \frac{A_{-}}{A_{+}}\left(-\lambda^{2 / 3} \eta\right) e^{i \lambda \psi(x, \eta)} d \eta \bmod \left(\lambda^{-\infty}\right), \\
w_{F}(x, \lambda) & \equiv \lambda^{\alpha+n / 4} \int g(x, \eta ; \lambda) e^{i \lambda \psi(x, \eta)} d \eta \bmod \left(\lambda^{-\infty}\right),
\end{aligned}
$$

where $\psi$ is a nondegenerate phase function and $\omega=\exp (2 \pi i / 3)$. For $\varrho=0$ we need to replace $A_{-} / A_{+}$by $A_{-}^{\prime} / A_{+}^{\prime}$. 


\section{The Geometry}

In order to understand the relation between the phase function in Proposition 1 and the geometry of the obstacle, $\partial K$, we shall now outline a slightly different construction of the phase function (compare [9]).

The general idea of such constructions is to parametrize the bicharacteristics by the distance to boundary rather than the time, as we are solving the Dirichlet rather than Cauchy problem. Nonuniqueness in such parametrization leads to a singularity in the phase which takes the simplest form in the case of strictly convex boundaries. To obtain the normal form for the singular part of the phase on the boundary, which plays the crucial role in the diffractive parametrix and here, we need the Melrose equivalence theorem for glancing hypersurfaces [2,9].

Let $p$ and $q$ be functions on $T^{*} X \backslash 0$ with independent differentials at $m \in$ $T^{*} X \backslash 0$. We denote by $P$ and $Q$ the hypersurfaces defined by $p$ and $q$ respectively. We say that $P$ and $Q$ are glancing at $m$ if

$$
\{p, q\}=0, \quad\{p,\{p, q\}\} \neq 0, \quad\{q,\{q, p\}\} \neq 0 .
$$

In our case we take $T^{*} X=T^{*} \mathbf{R}^{n+1}, q$ to be the defining function of $\partial K$, such that $\mathcal{O}=\mathbf{R}^{n} \backslash K=\{x: q(x)>0\}$, and $p=\tau^{2}-|\xi|^{2}$. The nondegeneracy conditions in (3) hold for all $m$ with $\{p, q\}=0$ if and only if $\partial K$ is strictly convex (see [5]).

The set of $m$ for which (3) holds is a hypersurface $L$ inside $P \cap Q$. The glancing situation can be described in terms of the following diagram:

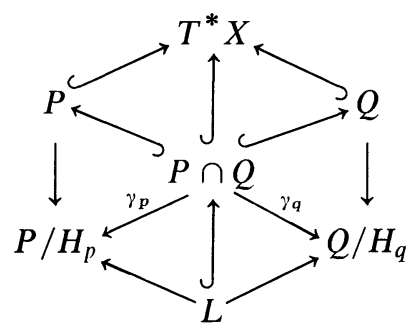

where $\gamma_{p}, \gamma_{q}$ are the natural projections:

$$
\begin{aligned}
& \gamma_{p}: P \rightarrow P / H_{p}, \\
& \gamma_{q}: Q \rightarrow Q / H_{q},
\end{aligned}
$$

in the diagram restricted to $P \cap Q$.

The equivalence theorem gives a conic neighbourhood of $(0,(1,0, \ldots, 0)) \in$ $T^{*} \mathbf{R}^{n+1} \backslash 0, \Gamma$, and a canonical transformation,

$$
\chi: \Gamma \rightarrow T^{*} X \backslash 0
$$

taking the (Friedlander) model glancing hypersurfaces:

$$
P_{F}=\left\{p_{F}=\xi_{n+1}^{2}-\xi_{1} \xi_{n}-x_{n+1} \xi_{1}^{2}=0\right\}, \quad Q_{F}=\left\{q_{F}=x_{n+1}=0\right\}
$$

to $P$ and $Q$ respectively.

By introducing normal geodesic coordinates we can assume that $Q=\left\{x_{n+1}\right.$ $=0\}$ with the time variable denotes by $x_{1}$. Thus the principal symbol becomes

$$
\xi_{1}^{2}-\left(\xi_{n+1}^{2}+r\left(x^{\prime}, x_{n+1}, \xi^{\prime}\right)\right) .
$$


Let $\mathscr{C}$ denote the usual wave relation:

$$
\begin{gathered}
\mathscr{C} \subset P \times P \subset T^{*} X \backslash 0 \times T^{*} \backslash 0, \\
\mathscr{C}=\left\{(r, s): r, s \in P, \gamma_{p}(r)=\gamma_{p}(s)\right\} .
\end{gathered}
$$

We should note that the usual of the eikonal equation (related to the Cauchy problem) is obtained by observing that

$$
\mathscr{C} \ni((x, \xi),(\tilde{x}, \tilde{\xi})) \mapsto\left(x, \tilde{x}_{1}, \tilde{\xi}_{2}, \ldots, \tilde{\xi}_{n+1}\right)
$$

is a local diffeomorphism and using the appropriate parametrization of $\mathscr{C}$ [2, Theorem 21.2.18]. Here, we shall consider

$$
\mathscr{C}_{1} \subset T^{*} X \backslash 0 \times \gamma_{q_{F}}(\Gamma \cap Q) \subset T^{*} \backslash 0 \times T^{*} \mathbf{R}^{n} \backslash 0,
$$

where we identify $T^{*} \mathbf{R}^{n} \cong Q_{F} / H_{q_{F}}$ and define

$$
\mathscr{C}_{1}=\left\{(r, m): m \in \bigcap_{(r, s) \in \mathscr{C}, s \in Q} \mathscr{C}_{0}\left(\gamma_{q_{F}}\left(\chi^{-1}(s)\right)\right)\right\},
$$

where $\mathscr{C}_{0}$ is a square root of the model billiard ball map given by (14) below. Instead of (6) we shall consider

$$
\mathscr{C}_{1} \ni(r,(x, \xi)) \mapsto(\pi(r), \xi),
$$

where $(x, \xi) \in T^{*} \mathbf{R}^{n} \backslash 0$ and $\pi: T^{*} X \backslash 0 \rightarrow X$. As a submanifold in a conic neighbourhood of $(m,(0 ;(1,0, \ldots, 0))) \in T^{*} X \backslash 0 \times T^{*} \mathbf{R} \backslash 0, \mathscr{C}_{1}$ is Lagrangian and the map given by (7) is a projection into another Lagrangian, $W$ :

$$
W \subset T^{*} X \backslash 0 \times T^{*} \mathbf{R}^{n} \backslash 0 ; \quad W=N^{*}\left\{\pi\left(T^{*} \mathbf{R}^{n}\right)\right\} .
$$

The map $\mathscr{C}_{1} \rightarrow W$ defined by (7) has a simple fold along $\left\{\xi_{n}=0\right\}$.

The local coordinates on the Lagrangian $W$ are given by $(x, \xi), x \in X$ and $\xi$ in the fibers of $\Gamma$. By modifying the proof of Theorem 21.4.1 in [2] (taking $W$ instead of the base), we obtain a phase function $\phi$,

$$
\phi(x, \xi)=\theta(x, \xi) \pm \frac{2}{3}(-\zeta(x, \xi))^{3 / 2}
$$

with $\theta$ and $\zeta$ smooth and such that

$$
\mathscr{C}_{1}=\left\{\left(\left(x, \phi_{x}^{\prime}\right),\left(\phi_{\xi}^{\prime}, \xi\right)\right)\right\}
$$

We could also use a smooth, homogeneous parametrization by introducing

$$
\tilde{\phi}(x, \xi, \tau)=-\frac{1}{3} \xi_{1}\left(\frac{\tau}{\xi_{1}}\right)^{3}-\tau \xi_{1}^{-2 / 3} \zeta(x, \xi)+\theta(x, \xi),
$$

(note that $\xi$ is in a conic neighbourhood of $(1,0, \ldots, 0)$ ). In terms of $\tilde{\phi}, \mathscr{C}_{1}=$ $\left\{\left(x, \tilde{\phi}_{x}^{\prime} ; \tilde{\phi}_{\xi}^{\prime}, \xi\right): \tilde{\phi}_{\tau}=0\right\}$.

As in Theorem 25.3.11 of [2] (see [7,9]) this immdiately motivates the presence of Airy functions. One should however remark that in studying boundary problems one leaves the calculus of Fourier Integral Operators as one uses the normalized non-tempered Airy functions. 
The main point in using the equivalence theorem in the definition of $\mathscr{C}_{1}$ is that it provides the normal form for $\zeta$ restricted to the boundary,

$$
\begin{gathered}
B=\partial K \times \mathbf{R}, \\
\zeta_{0}(\xi)=\left.\zeta(x, \xi)\right|_{B \times \mathbf{R}^{n}}=-\xi_{1}^{-1 / 3} \xi_{n} .
\end{gathered}
$$

The geometric object obtained from the bicharacteristic foliation of $P$ and $Q$ is the billiard ball map defined on $T^{*} B \backslash 0$,

$$
\begin{aligned}
& \Sigma=\left\{\left(z, \delta^{ \pm}(z)\right)\right\} \subset T^{*} B \backslash 0 \times T^{*} B \backslash 0, \quad T^{*} B \backslash 0 \cong Q / H_{q}, \\
& \Sigma=\left\{\left(z_{1}, z_{2}\right): z_{i}=\gamma_{q}\left(\tilde{z}_{i}\right), \tilde{z}_{i} \in P, \gamma_{p}\left(\tilde{z}_{1}\right)=\gamma_{p}\left(\tilde{z}_{2}\right)\right\} .
\end{aligned}
$$

In the model case the billiard ball map takes a particularly simple form:

$$
\delta_{0}^{ \pm}(x, \xi)=\left(x_{1} \mp \frac{2}{3}\left(\frac{\xi_{n}}{\xi_{1}}\right)^{3 / 2}, x_{2}, \ldots, x_{n} \pm 2\left(\frac{\xi_{n}}{\xi_{1}}\right)^{1 / 2}, \xi\right)
$$

Taking $\Gamma_{\partial}=\left\{(x, \xi):\left(x, 0, \xi, \xi_{n+1}\right) \in \Gamma\right\}$ we can use $\chi$ to obtain $\chi_{\partial}$ :

$$
\chi_{\partial}: \Gamma_{\partial} \rightarrow T^{*} B \backslash 0,
$$

conjugating $\delta^{ \pm}$to the normal form:

$$
\chi_{\partial}^{-1} \delta^{ \pm} \chi_{\partial}=\delta_{0}^{ \pm}
$$

The construction above shows that the phase function $\theta_{0}=\left.\theta\right|_{B \times \mathbf{R}^{n}}$ generates $\chi_{\partial}$, i.e.

$$
\chi_{\partial}:\left(x,\left(\theta_{0}\right)_{x}^{\prime}\right) \mapsto\left(\left(\theta_{0}\right)_{\xi}^{\prime}, \xi\right)
$$

The other, singular, component of our phase $\phi$ restricted to the boundary integrates the billiard ball map, as easily seen from (10) and (9):

$$
\delta_{0}^{ \pm}=\exp \left(H_{ \pm \frac{4}{3}\left(-\zeta_{0}\right)^{3 / 2}}\right) .
$$

If for $(y, \eta) \in T^{*} B$ we define

$$
\zeta_{\#}(y, \eta)=\zeta_{0}\left(\xi\left(\chi_{\partial}^{-1}(y, \eta)\right)\right),
$$

then, since $\chi_{\partial}$ is a symplectomorphism, we obtain

$$
\delta^{ \pm}=\exp \left(H_{ \pm \frac{4}{3}\left(-\zeta_{\sharp}\right)^{3 / 2}}\right) .
$$

Thus this geometrically defined function (via its relation with the billiard ball map) establishes the essential connection between the normal form and the geometry of the scatterer.

Rather naturally, for both Proposition 1 ([8]) and the relation between the variables in the integral expression for $w, w_{F}$ we need to understand the way $\tilde{\Lambda}_{D}$, the free flow-out of $\Lambda$, behaves under $\chi$. Fortunately the rigidity in the equivalence theorem comes only from intertwining the billiard ball maps and as in $[7,8,11]$ we can follow Melrose in using the slightly refined version of the equivalence theorem which puts $\tilde{\Lambda}_{D}$ into normal form. 
More precisely we shall use Theorem 4.10 of [7] applied to the following situation (just slightly different from the application in [7] - see [8]). Let us define the following folding canonical relation ${ }^{2}$ :

$$
\mathscr{C}^{\prime \prime} \subset T^{*} B \backslash 0 \times T^{*} \mathcal{O} \backslash 0, \quad \mathscr{C}^{\prime \prime}=\left\{\left(z_{1}, z_{2}\right): \gamma_{q}\left(\left.\exp H_{p}\left(z_{1}\right)\right|_{Q}\right)=z_{2}\right\} .
$$

We have $\Lambda \subset T^{*} \mathscr{O} \hookrightarrow \mathscr{C}^{\prime \prime}$ intersecting the singular set of $\mathscr{C}^{\prime \prime}$ transversally and thus we can locally find

$$
\begin{aligned}
& \chi_{1}: T^{*} \mathcal{O} \backslash 0 \rightarrow T^{*} \mathbf{R}^{n}, \\
& \chi_{2}: T^{*} B \backslash 0 \rightarrow T^{*} \mathbf{R}^{n},
\end{aligned}
$$

such that $\left(\chi_{2} \times \chi_{1}\right)\left(\mathscr{C}^{\prime \prime}\right)=\mathscr{C}_{0}$, where $\mathscr{C}_{0}$ is the model folding canonical relation (really the square root of the billiard ball map):

$$
\begin{aligned}
& \left((x, \xi ; y, \eta): x_{i}=y_{i}, 1<i<n, \xi=\eta,\left(y_{1}-x_{1}\right)\right. \\
& \left.\quad=\frac{1}{3}\left(x_{n}-y_{n}\right)^{3},\left(x_{1}-x_{1}\right)^{2}=-\frac{\xi_{n}}{\xi_{1}}\right\} .
\end{aligned}
$$

Simultaneously $\Lambda$ is put to normal form:

$$
\chi_{1}(\Lambda)=N^{*}\left\{x_{1}=x_{n}=0\right\} .
$$

The canonical transformation $\chi_{2}$ intertwines the billiard ball maps and the equivalence function $\chi$ can be constructed (actually the construction starts with showing the existence of $\chi_{\partial}-$ see Sect. 3.5-3.6 of [9]) so that $\chi_{\partial}=\chi_{2}$. If we define

$$
\left(\tilde{\Lambda}_{D}\right)_{h}=\left\{z \in \tilde{\Lambda}_{D}, \exp H_{p}(z) \cap Q \neq \emptyset\right\},
$$

then from (10) and (11) we obtain

$$
\left(\tilde{\Lambda}_{D}\right)_{h}=\chi\left[\mathscr{C}_{2}\left(\Lambda_{0} \cap\left\{\xi_{n} \geq 0\right\}\right)\right],
$$

where $\mathscr{C}_{2}$ is the model wave relation parametrized by

$$
\begin{aligned}
\phi_{0} & =x \cdot \xi \pm \frac{2}{3} \xi_{1}^{-1 / 2}\left(\xi_{n}+\xi_{1} x_{n+1}\right)^{3 / 2}, \\
x & =\left(x_{1}, \ldots, x_{n}\right), \quad \xi=\left(\xi_{1}, \ldots, \xi_{n}\right),
\end{aligned}
$$

i.e. $\mathscr{C}_{2}=\left\{\left(x,\left(\phi_{0}\right)_{x}^{\prime} ;\left(\phi_{0}\right)_{\xi}^{\prime}, \xi\right)\right\}$. Consequently

$$
\left(\tilde{\Lambda}_{D}\right)_{h}=\mathscr{C}_{1}\left(\Lambda_{0} \cap\left\{\xi_{n} \geq 0\right\}\right) .
$$

Both sides are Lagrangians with boundaries but one could actually modify $\chi$ to obtain (15) without $h$ and $\xi_{n} \geq 0$ on the left- and right-hand sides respectively.

We shall now study the phase in Proposition 1, and we only need to consider $w_{F}$. For that we can use Lemma 3.1 of [11], with immediate motivation from (8):

$$
u_{F}(t, x)=\int\left[g(x, \xi) A i(\zeta)+h(x, \xi) A i^{\prime}(\zeta)\right] e^{i\left(\theta_{1}(x, \xi)+t \xi_{1}\right)} \hat{f}(\xi) d \xi
$$

where because of translation invariance we can have

$$
\chi^{*} \xi_{1}=\xi_{1}, \quad \theta(t, x, \xi)=\theta_{1}(x, \xi)+t \xi_{1},
$$

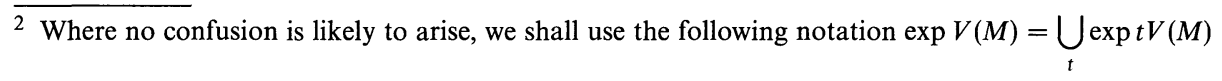


and where $f \in I_{\mathrm{cl}}^{\alpha+\frac{1}{6}}\left(\mathbf{R}^{n}, N^{*}\left\{x_{1}=x_{n}=0\right\}\right.$ ), because of (15) (compare (3.8) of [8] and Sect. 4 of [11]), $g \in S_{\mathrm{cl}}^{0}, h \in S_{\mathrm{cl}}^{-1 / 3}$. With $a$ the symbol of $f, f(x)=$ $a\left(x^{\prime \prime}, D\right) \delta\left(x_{1}\right) \delta\left(x_{n}\right), x^{\prime \prime}=\left(x_{2}, \ldots, x_{n-1}\right)$ we have

$w_{F}(x, \lambda)=\int\left[g A i\left(\zeta\left(x ; \lambda, 0, \xi_{n}\right)\right)+h A i^{\prime}\left(\zeta\left(x ; \lambda, 0, \xi_{n}\right)\right)\right] e^{i \theta_{1}\left(x, \lambda, 0, \xi_{n}\right)} a_{1}\left(x, \lambda, 0, \xi_{n}\right) d \xi_{n}$,

with $a_{1} \in S_{\mathrm{cl}}^{\alpha+n / 4-5 / 6}$. Using the integral representation of the Airy function and changing variables $\xi_{n}=\lambda \eta$ we obtain

$$
w_{F}(x, \lambda)=\lambda^{4 / 3} \int\left[g+\lambda^{-1 / 3} \tau h\right] a_{1} e^{i \lambda\left[-\frac{1}{3} \tau^{3}-\tau \zeta(z, 1,0, \eta)+\theta_{1}(x, 1,0, \eta)\right]} d \tau d \eta .
$$

By (9) and the diffractive condition $\{p,\{p, q\}\}(m)>0$,

$$
\zeta=-\xi_{1}^{-1 / 3}\left(\xi_{n}+\varrho(x, \xi)\right), \quad \varrho=0,\left.\quad \partial_{\nu} \zeta\right|_{\partial K \times \mathbf{R}^{n}}>c \xi_{1}>0 .
$$

Thus if $\left|\xi_{n}\right|<c^{\prime} \delta \xi_{1}$ and $d(x, \partial K)>\delta$, then $\zeta<-c^{\prime \prime} \xi^{2 / 3}$. That allows us to apply the stationary phase method in the $\tau$ variable in (18), which amounts to replacing $A i$ and $A i^{\prime}$ in (17) by their asymptotic expansions. Since near the shadow $\Gamma_{+}$only the outgoing term in $A i, A_{+}$contributes, the phase we obtain is

$$
\psi(x, \eta)=-\frac{2}{3}(-\zeta(x, 1,0, \eta))^{3 / 2}+\theta_{1}(x, 1,0, \eta) .
$$

An application of the Morse lemma gives

$$
\psi(x, \eta(x, \sigma))=\bar{\psi}(x)+\frac{1}{2} h(x)(\sigma-\bar{\sigma}(x))^{2}, \quad \bar{\psi}(x)=x \cdot \omega+a,
$$

where $\bar{\sigma}(x)=-\bar{\eta}(x)$ is the critical value of $\psi$,

$$
\eta(x, \sigma)=-\sigma+O\left((\sigma-\bar{\sigma}(x))^{2}\right) .
$$

The function $h$ is smooth for $d(x, \partial K)>\delta$ and (see (6.13) of [11]) it takes the form $h=\bar{d} / 2 \bar{t}$, where $\bar{d}, \bar{t}$ are smooth with $\left.\bar{t}\right|_{\partial K}$ defining $\Phi \cap \partial K$, the shadow boundary on $\partial K$. Also, $\bar{t}(x)>0$ and $\bar{d}(x)<0$ for $x$ close to $\Gamma_{+}$in $d(x, \partial K)>\delta$. Thus

$$
x \in \Gamma_{+} \Rightarrow h(x)>0 .
$$

Since by (15)

$$
\bar{\eta}(x)=\chi^{*}\left(\xi_{1}^{-1} \xi_{n}\right)(x, \omega), \quad \Lambda_{D}=\{(x, \omega)\} .
$$

$\bar{\eta}$ simply vanishes on $\Gamma$ and thus

$$
\bar{\eta}(x)=c(x) \bar{s}(x), \quad c \neq 0 .
$$

As we shall see from the expansions in Sect. 4 the value of $c$ on $\Gamma$ determines the size of the shift. The relation of $c$ to the geometry of $\partial K$ is given by the following proposition, closely related to Lemma 5.12 of [5].

Proposition 2. The value of $c$ defined by (22) on the shadow boundary is

$$
\left.c(x)\right|_{\Gamma}=[2 \alpha(y(x), \omega)]^{1 / 3},
$$

where $y(x)=L_{x} \cap \partial K$, with $L_{x}=\{x+t \omega: t \in \mathbf{R}\}$, the glancing ray through $x$, and where $\alpha$ is the directional curvature of $\partial K$ at $y(x)$ in the direction $\omega$. 
Proof. Let us first observe that $\left.c(x)\right|_{\Gamma}$ is constant on the rays in $\Gamma$. Indeed, we can consider $c \mid \Gamma$ as a $\operatorname{limit} \lim _{y \rightarrow x} \bar{\eta}(y) / \bar{s}(y), y \notin \Gamma$. But $\bar{\eta}$ is constant on rays in $\Lambda_{D}$ by construction and for $\Lambda_{D}=\{(x, \omega)\}$, the rays stay equidistant to $\Gamma^{3}$. As $\chi^{*} \xi_{1}=\xi_{1}$ we obtain from (12) and (21),

$$
x \in \partial K \Rightarrow \bar{\eta}=-\lambda^{-2 / 3} \zeta_{\#}\left(\gamma_{q}(x, a+x \cdot \omega, \lambda \omega, \lambda)\right) .
$$

Let $y \in \partial K$ be a glancing point for $\Lambda_{D}$ and $L$ the glancing ray through $y$. Let us consider $\tilde{L}$, a ray in the direction $\omega$ coplanar with $L$ and the normal to $\partial K$ at $y$, $v_{y}$. and

Let $x \in \tilde{L} \cap \partial K$ be the second point of intersection of $\tilde{L}$ and $\partial K$ (see Fig. 2)

$$
\xi=\xi\left(\gamma_{q}(x, \omega)\right), \quad \gamma_{q}: T_{\partial K}^{*} \mathbf{R}^{n} \rightarrow T^{*} \partial K .
$$

If $\alpha(y, \omega)$ is the directional curvature of $\partial K$ at $y$ in the direction $\omega$, when the distance, $s$, between $L$ and $\tilde{L}$ can be considered a function of $|\xi|$ :

$$
s(|\xi|)=-[\alpha(y, \omega)]^{-1}(1-|\xi|)+O\left((1-|\xi|)^{2}\right) .
$$

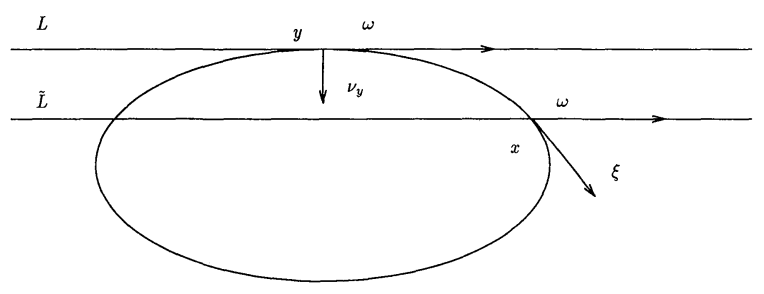

Fig. 2

Comparing with (23) and rescaling in $\tau$ we obtain

$$
c(y)=\alpha(y, \omega) \lim _{\tau \downarrow 1}(\tau-1)^{-1} \zeta_{\#}\left(\gamma_{q}(x, x \cdot \omega+a ; \omega / \tau, 1)\right) .
$$

Thus we need to evaluate the limit (except for the factors of 2 this is essentially done in Lemma 5.12 of [5]). For this we observe that

$$
\lim _{\tau \downarrow 1}(\tau-1)^{-1} \zeta_{\sharp}\left(\gamma_{q}(x, x \cdot \omega+a ; \omega / \tau, 1)\right)=\omega \cdot \partial_{\xi} \zeta(y, t, \omega, 1), \quad(y, \xi) \in T^{*} \partial K .
$$

The billiard ball map gives a point $\tilde{y}$ at which the ray from $y$ in the direction $\omega / \tau+\left(1-\tau^{-2}\right)^{1 / 2} v_{y}$ hits the boundary $\partial K$ :

$$
\tilde{y}=\left[\frac{4}{3}(-\zeta(t, y, 1, \omega / \tau))^{3 / 2}\right]_{\xi}^{\prime}+O((\tau-1)) .
$$

On the other hand (see Fig. 3)

$$
\tilde{y}=\frac{2}{\alpha}\left[\frac{\omega}{\tau}+\left(1-\tau^{-2}\right)^{1 / 2} v_{y}\right]\left(1-\tau^{-2}\right)^{1 / 2}+O((\tau-1)) .
$$

\footnotetext{
3 This is where we use the plane wave assumption. Otherwise we would get a variable factor in $c$ depending only on $A$ or, in a more general setting on a variable metric
} 


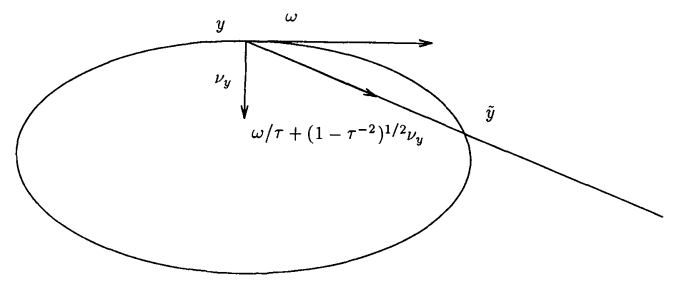

Fig. 3

Using (24) this easily leads to

$$
\lim _{\tau \downarrow 1}(\tau-1)^{-1} \zeta_{\sharp}\left(\gamma_{q}(x, x \cdot \omega+a ; \omega / \tau, 1)\right)=2^{1 / 3}[\alpha(y, \omega)]^{-2 / 3},
$$

concluding the proof.

\section{Asymptotic Expansions}

For the expansions of the free wave we only need to use the stationary phase method. If we define

$$
p_{F}(x, \lambda)=\lambda^{-\alpha-n / 4} e^{-i \lambda x \cdot \omega} w_{F}(x, \lambda),
$$

and $g_{0}(x, s)=g(x, s, \lambda)+O\left(\lambda^{-1}\right)$, then

$$
p_{F}(x, \lambda)=\left(\frac{2 \pi i}{\lambda h(x)}\right)^{1 / 2} e^{-\frac{\pi l}{4}}\left[g_{0}(x, \bar{\eta}(x))+O\left(\lambda^{-1}\right)\right] .
$$

Of course, as in the case considered by Keller and Rubinow, $w_{F}$ might be given explicitly $(\exp (i \lambda x \cdot \omega))$, but the main point here is the comparison of the coefficients in the expansions of $w$ and $w_{F}$. Let us now consider $p(x, \lambda)$, $w(x, \lambda)=\lambda^{\alpha+n / 4} e^{i \lambda \bar{\psi}(x)} p(x, \lambda)$,

$$
p(x, \lambda)=-\omega^{2} \int g(x, \eta, \lambda) \frac{A_{-}}{A_{+}}\left(\lambda^{2 / 3} \eta\right) e^{\frac{1}{2} i \lambda h(x)(\sigma-\bar{\sigma}(x))^{2}} d \sigma,
$$

with $\eta=\eta(x, \sigma)$ given by the Morse lemma.

The behaviour of the Airy quotient is of course crucial (cf. Lemma 4.2 of [11]):

$$
\frac{A_{-}}{A_{+}}(t) \sim \begin{cases}-\exp (2 \pi i / 3)+O\left(t^{-\infty}\right) & t \rightarrow+\infty \\ e^{i \frac{4}{3}(-t)^{3 / 2}} \sum_{j \geq 0} \beta_{j} t^{-3 j / 2} & t \rightarrow-\infty\end{cases}
$$

As in $[8,11]$ this suggests a partition of $p$ according to the behaviour of $A_{-} / A_{+}$, which physically corresponds to partition into direct, reflected and diffracted waves and has also a microlocal characterization (see [11,6]). Unlike there however we are not interested in the precise uniform expansions of the diffracted wave, in particular the nature of the cancellation in the shadow, but only in the size of the leading term of $p$ compared to $p_{F}$. Both the expansions and the computation of the leading term are much easier if the integral representing $p$ 
is partitioned in a way allowing a certain overlap between the terms. Thus we define:

$$
\begin{aligned}
& u_{\#}(s)=-\omega^{2} Y_{+}(s)\left(\frac{A_{-}}{A_{+}}(s)+\omega\right)=\omega Y_{+}(s) \frac{A i}{A_{+}}(s), \\
& \Phi_{\#}(s)=-\omega^{2} Y_{+}(s) \frac{A_{-}}{A_{+}}(-s)=\Phi(s)+u_{b},
\end{aligned}
$$

where $\Phi=\Phi_{\sharp} \chi$ with $\chi \in C^{\infty}$ supported on the positive half axis and identically 1 for $s>1$. Here $Y_{+}$denotes the Heavyside function on the positive half axis.

Thus we define $p_{i}$ for $i=1,2,3,4, p=\sum_{i=1} p_{i}$ :

$$
\begin{aligned}
& p^{1}(x, \lambda)=\int_{0}^{\infty} g(x, \eta, \lambda) e^{\frac{1}{2} i \lambda h(x)(\sigma-\bar{\sigma}(x))^{2}} d \sigma, \\
& p^{2}(x, \lambda)=\int_{0}^{\infty} u_{\#}\left(\lambda^{2 / 3} \eta\right) g(x, \eta, \lambda) e^{\frac{1}{2} i \lambda h(x)(\sigma-\bar{\sigma}(x))^{2}} d \sigma, \\
& p^{3}(x, \lambda)=\int \Phi\left(-\lambda^{2 / 3} \eta\right) g(x, \eta, \lambda) e^{\frac{1}{2} i \lambda h(x)(\sigma-\bar{\sigma}(x))^{2}} d \sigma, \\
& p^{4}(x, \lambda)=\int u_{b}\left(-\lambda^{2 / 3} \eta\right) g(x, \eta, \lambda) e^{\frac{1}{2} i \lambda h(x)(\sigma-\bar{\sigma}(x))^{2}} d \sigma .
\end{aligned}
$$

As remarked above there is an overlap in supports between the terms $p^{1}$ and $p^{2}$ and in the singularity at 0 for $p^{2}$ and $p^{4}$. In effect the integrals do not vanish rapidly $\left(O\left(\lambda^{-\infty}\right)\right)$ in the shadow as $\lambda$ tends to inifinity, but have rather straightforward expansions in $\lambda^{-1 / 2}$ with terms cancelling in the shadow. This however suffices for the investigation of the size of $p$.

The integrals of the form $p^{1}$ go back to Fresnel and have made frequent appearance in the nonrigorous study of diffraction based on the Kirchhoff approximation (assuming that the field is essentially zero in the shadow). For the convenience of the reader we shall present the expansion of a more general integral which was provided by Melin [4] (compare [2], Theorem 7.7.17). Let

$$
J_{t}(w)=\int_{s \geq 0, y \in \mathbf{R}^{n}} e^{i t G(s, y, w)} h(s, y, w) d s d y, \quad t>0, \quad w \in \mathbf{R}^{n},
$$

where $G, h \in C^{\infty}\left(\overline{\mathbf{R}}_{+} \times \mathbf{R}^{2 n}\right)$ and

$$
\partial_{s} G(0)=\partial_{y} G(0)=0, \quad G_{s s}^{\prime \prime}(0) \neq 0, \quad \operatorname{det} G_{y y}^{\prime \prime} \neq 0 .
$$

Let us also define the "Fresnel integral":

$$
\Lambda_{+}(r)=(2 \pi)^{-1 / 2} e^{-i \frac{\pi}{4}} \int_{-\infty}^{r} e^{i \frac{z^{2}}{2}} d z
$$

and $\Lambda_{-}(r)=\overline{\Lambda_{+}(r)}$. We should also note that

$$
\Lambda_{+}(r)=\left\{\begin{array}{ll}
1+O\left(r^{-1}\right) & r \rightarrow+\infty \\
O\left(r^{-1}\right) & r \rightarrow-\infty
\end{array},\right.
$$

and $\Lambda_{ \pm}(0)=\frac{1}{2}$. Now we have [4]: 
Lemma 1. For $J_{t}$ defined by (30) and $G$ satisfying (31) let $(s(w), y(w), w)$ be the unique critical point near the origin for $(s, y) \mapsto G(s, y, w)$. Set

$$
\Lambda_{G}=\left\{\begin{array}{ll}
\Lambda_{+} & \text {if } G_{s s}^{\prime \prime}>0 \\
\Lambda_{-} & \text {if } G_{s s}^{\prime \prime}<0
\end{array} .\right.
$$

Then

$$
\left.\left|J_{t}(w)-(2 \pi)^{\frac{n+1}{2}} t^{-\frac{n+1}{2}} e^{i \frac{\pi \sigma}{4}} e^{i t \bar{G}(w)} \Lambda_{G}\left(t^{1 / 2} \tilde{S}(w)\right)\right| \operatorname{det} \bar{G}^{\prime \prime}(w)\right|^{-1 / 2} \mid \leq C t^{-\frac{n+2}{2}},
$$

where $\bar{G}(w)=G(s(w), y(w), w), \bar{G}^{\prime \prime}=G^{\prime \prime}(s(w), y(w), w)$, the second derivative with respect to $y$ and $s, \sigma=\operatorname{sign} \bar{G}^{\prime \prime}$, and

$$
\tilde{s}(w)=\operatorname{sign}(s(w))|2(G(0, y(0, w), w)-G(s(w), y(w), w))|^{1 / 2} .
$$

To understand the behaviour of $p^{1}$ we only need the simplest version of this lemma, and we just need to recall that the sign of $h$ is negative, (20).

Proposition 3. For $p^{1}$ given by (26) we have

$$
p^{1}(x, \lambda)=\left(\frac{2 \pi}{\lambda|h(x)|}\right)^{1 / 2} e^{-\frac{\pi t}{4}} \Lambda_{-}\left(\lambda^{1 / 2}|h(x)|^{1 / 2} \bar{\sigma}(x)\right) g(x, \bar{\sigma}(x))+O\left(\lambda^{-1}\right) .
$$

The properties of the $p^{2}$ and $p^{4}$ terms are similar and are described in the following

Proposition 4. Let $q$ be given by

$$
q(x, \lambda)=\int Y_{ \pm} u\left(\lambda^{2 / 3} \eta\right) a(x, \eta) e^{\frac{1}{2} i \lambda h(x)(\sigma-\bar{\sigma}(x))^{2}} d \sigma
$$

with $u \in \mathscr{S}(\mathbf{R})$ and $a \in C_{0}^{\infty}\left(\mathbf{R}^{2}\right)$. Then

$$
q(x, \lambda)=e^{\frac{1}{2} i \lambda h(x) \bar{\tau}(x)^{2}} \lambda^{-2 / 3} a(x, 0) F\left(\lambda^{1 / 3} h(x) \bar{\tau}(x)\right)+O\left(\lambda^{-1}\right)+O\left(\lambda^{-2 / 3} \bar{\sigma}(x)^{2}\right),
$$

where $\bar{\tau}(x)=\bar{\sigma}(x)\left(1+c_{2}(x) \bar{\sigma}(x)\right)$ and

$$
F(\tau)=\int Y_{ \pm}(-t) u(-t) e^{i t \tau} d t
$$

Proof. We shall first introduce a new variable $\tau=\tau(x, \sigma), \sigma=\sigma(x, \tau)$, such that $\eta(x, \sigma(x, 0))=0$ and $\sigma(x, \tau)-\bar{\sigma}(x)=\tau-\bar{\tau}(x)$. This is obtained easily from the implicit function theorem which, using (19), also yields $\bar{\tau}(x)=\bar{\sigma}(x)\left(1+c_{2}(x) \bar{\sigma}(x)\right)$. Indeed, we can introduce new coordinates in $\mathbf{R}^{n}, x \mapsto y$ so that $\bar{\sigma}(x)=y_{1}$. Then $\eta(x, s)=\tilde{\eta}(y, s)$ and we are solving $\tilde{\eta}\left(y, y_{1}-\tilde{\tau}(y)\right)=0$, where $\bar{\tau}(x)=\tilde{\tau}(y(x))$. Vanishing of $y_{1}$ implies vanishing of $\tilde{\tau}(y)$ so that $\left.\left(\partial_{y_{1}} \tilde{\eta}\right)(y,-\tilde{\tau}(y))\right|_{y_{1}=0}=0$ and by the implicit function theorem $\left.\partial_{y_{1}} \tilde{\tau}\right|_{y_{1}=0}=1$.

With the new variable and $\varrho(x, \tau)=\eta(x, \sigma(x, \tau))$ the integral becomes:

$$
\int Y_{ \pm}(\sigma(x, \tau)) u\left(\lambda^{2 / 3} \varrho(x, \tau)\right) a_{1}(x, \varrho(x, \tau)) e^{\frac{1}{2} h(x)(\tau-\bar{\tau})^{2}} d \tau,
$$

where $a_{1}(x, 0)=a(x, 0)$. Now, we expand

$$
\varrho(x, \tau)=\tau \varrho_{0}(x, \tau), \quad \varrho(x, \tau)=-\tau+\tau^{2} \varrho_{1}(x, \tau),
$$


and introduce $t=\lambda^{2 / 3} \tau$ which gives:

$$
\begin{aligned}
& \lambda^{-2 / 3} \int Y_{ \pm}\left(\sigma\left(x, \lambda^{-2 / 3} t\right)\right) u\left(-t+\lambda^{-2 / 3} t^{2} \varrho_{1}\left(x, \lambda^{-2 / 3} t\right)\right) \\
& a_{1}\left(x, \lambda^{-2 / 3} t \varrho_{0}\left(x, \lambda^{-2 / 3} t\right)\right) e^{\frac{1}{2} i \lambda h(x) \bar{\tau}(x)^{2}} e^{\frac{1}{2} i \lambda^{-1 / 3} h(x) t^{2}} e^{i h(x) t\left(\lambda^{1 / 3} \bar{\tau}(x)\right)} d t .
\end{aligned}
$$

If we take the support of $g$ in Proposition 1 sufficiently close to 0 (which we may as we are interested if $x$ close to $\left.\Gamma_{+}\right)$we can assume that $\left|-\tau+\tau^{2} \varrho_{1}(x, \tau)\right|>c|\tau|$. Thus we can expand $u$ and $a_{1}$ in Taylor series around $-t$ and 0 respectively, absorbing the powers of $t$ into the Schwartz term $u$. This gives:

$$
e^{\frac{1}{2} i \lambda h(x) \bar{\tau}(x)^{2}} \lambda^{-2 / 3} \int Y_{ \pm}\left(\sigma\left(x, \lambda^{-2 / 3} t\right)\right) u(-t) a_{1}(x, 0) e^{\frac{1}{2} i \lambda^{-1 / 3} h(x) t^{2}} e^{i h(x) t\left(\lambda^{1 / 3} \bar{\tau}(x)\right)} d t+O\left(\lambda^{-4 / 3}\right) .
$$

Now we can expand $\exp \left(\frac{1}{2} i \lambda^{-1 / 3} h(x) t^{2}\right)$ into Taylor series, again using the rapid decay of $u$. Finally we observe that $\left|\operatorname{supp}_{\tau}\left(Y_{ \pm}(\sigma(x, \tau))-Y_{ \pm}(\tau)\right)\right|=O\left(\bar{\sigma}(x)^{2}\right)$, since $\sigma(x, \tau)=\tau+O\left(\bar{\sigma}(x)^{2}\right)$. This gives the desired expansion of $q$

We are left with $p^{3}$ given by $(28)$ - here the analysis is the same as in $[8,11]$. By Lemma 4.2 or [11],

$$
\hat{\Phi}(t)=e^{-\frac{1}{12} i t^{3}} \Psi(t), \quad \Psi \in S_{p h g}^{1 / 2}(\mathbf{R}), \quad \Psi(t) \sim\left\{\begin{array}{ll}
\sum_{j \geq 0} \beta_{j} t^{1 / 2-3 j} & t \rightarrow+\infty \\
O\left(t^{-\infty}\right) & t \rightarrow-\infty
\end{array} .\right.
$$

Using this $p^{3}$ becomes

$$
p^{3}(x, \lambda)=\frac{1}{2 \pi} \lambda^{1 / 3} \int g(x, \eta, \lambda) \Psi\left(\lambda^{1 / 3} t\right) e^{i \lambda\left(-\frac{1}{12} t^{3}-t \eta+\frac{1}{2} h(x)(\sigma-\bar{\sigma}(x))^{2}\right)} d \sigma d t .
$$

The stationary phase method is applicable to this integral. Let us denote the phase by $\psi_{1}$ and the critical values of $(x, \sigma, t) \mapsto \psi_{1}(x, \eta(x, \sigma), t)$ by $\sigma_{+}(x)$ and $t_{+}(x)$. With $\bar{\psi}_{1}$ and $\bar{\psi}_{1}^{\prime \prime}$ the critical values of the phase and the Hessian we obtain:

$$
p^{3}(x, \lambda)=\lambda^{-2 / 3}\left|\operatorname{det} \bar{\psi}_{1}^{\prime \prime}(x)\right|^{-1 / 2} e^{i \lambda \bar{\psi}_{1}(x)}\left[g\left(x, \sigma_{+}(x)\right) \Psi\left(\lambda^{1 / 3} t_{+}(x)\right)+O\left(\lambda^{-1 / 3}\right)\right] .
$$

We need to understand the behaviour of $\sigma_{+}, t_{+}$and $\bar{\psi}$ near $\Gamma_{+}$. For this we have (compare Sect. 4 of [11]) the following straightforward

Lemma 2. For $x$ close to $\Gamma_{+}$we have:

$$
\begin{aligned}
t_{+}(x) & =\bar{\sigma}(x)+O\left(\bar{\sigma}(x)^{2}\right), \quad \sigma_{+}(x)=-\frac{1}{4} \sigma(x)^{2}+O\left(\bar{\sigma}(x)^{3}\right), \\
\bar{\psi}_{1}(x) & =O\left(\bar{\sigma}(x)^{3}\right), \quad \operatorname{det} \bar{\psi}_{1}^{\prime \prime}(x)=-1+O(\bar{\sigma}(x)) .
\end{aligned}
$$

Using this in (33) we obtain:

\section{Proposition 5.}

$$
p^{3}(x, \lambda)=\lambda^{-2 / 3}\left[g(x, 0) \Psi(0)+O\left(\lambda^{-1 / 3}\right)+O\left(\lambda^{1 / 3} \bar{\sigma}(x)\right)+O\left(\lambda \bar{\sigma}(x)^{3}\right)\right] .
$$

We can now give

Proof of Theorem 1. We need to add all the terms $p^{i}$ and compare the result to $p_{F}$. We can assume here that

$$
\left|\lambda^{2 / 3} \bar{\sigma}(x)\right| \leq C
$$


for that where $x$ in the theorem is. Let us also recall that

$$
\Lambda_{-}(0)=\frac{1}{2} ; \quad \Lambda_{-}^{\prime}(0)=(2 \pi)^{-1 / 2} e^{\frac{i \pi}{4}} .
$$

Thus in (34) we have

$$
p^{1}(x, \lambda)=\frac{1}{2}\left(\frac{2 \pi}{\lambda|h(x)|}\right)^{1 / 2} e^{-\frac{\pi l}{4}} g(x, 0)+\bar{\sigma}(x) g(x, 0)+O\left(\lambda^{-1}\right) .
$$

Using Propositions 4 and 5 and comparing with (25) we obtain for $x$ in (34):

$$
\begin{aligned}
p(x, \lambda)= & p_{F}(x, \lambda)\left[\frac{1}{2}+\left(\frac{\lambda|h(x)|}{2 \pi}\right)^{1 / 2} e^{\frac{\pi l}{4}}\left(\bar{\sigma}(x)-\lambda^{-2 / 3}\left(F_{\#}(0)+F_{b}(0)+\Psi(0)\right)\right)\right] \\
& +O\left(\lambda^{-1}\right)
\end{aligned}
$$

where

$$
F_{\#}(0)=\int Y_{+} u_{\#}(t) d t, \quad F_{b}(0)=\int Y_{-} u_{b}(-t) d t .
$$

Going back to the definitions of $u_{\#}$ and $u_{b}$ we obtain:

$$
C_{1}=F_{\#}+F_{b}+\Psi(0)=e^{-\frac{2 \pi u}{3}} \int_{-\infty}^{0} \frac{A_{-}}{A_{+}}(t) d t+e^{-\frac{\pi t}{3}} \int_{0}^{\infty} \frac{A i}{A_{+}}(t) d t .
$$

If we now use Proposition 2 we obtain Theorem 1 in the case $\varrho=\infty$ with

$$
C(\infty)=2^{-1 / 3}\left(\mathfrak{R} C_{1}-\mathfrak{I} C_{1}\right)
$$

The modification in the $\varrho=0$ follows by replacing the Airy functions by their derivatives as in Proposition 1. For the general boundary condition (1) one needs to consider a quotient of linear combinations of the Airy function and its derivative. It is clear from the above that only the value of $\varrho$ at the glancing point is going to contribute to the shift of the shadow boundary.

Acknowledgements. I should like to thank Richard Melrose for encouraging me to write this paper.

\section{References}

1. Farris, M.: Egorov's theorem on a manifold with diffractive boundary. Commun. P.D.E. 6(6), 651-688 (1981)

2. Hörmander, L.: The analysis of linear partial differential operators. I-IV. Berlin, Heidelberg, New York: Springer 1983-1985

3. Keller, J.B., Rubinow, S.I.: Shift of the shadow boundary and scattering cross-section of an opaque object. J. Appl. Phys. 32(5), 814-820 (1961)

4. Melin, A.: On the asymptotic behaviour of some oscillatory integrals (unpublished)

5. Melrose, R.B.: Forward scattering by a convex obstacle. Commun. Pure Appl. Math. 23, 461-499 (1980)

6. Melrose, R.B., Sá Barreto, A., Zworski, M.: Semi-linear diffraction of conormal waves. Manuscript if preparation

7. Melrose, R.B., Taylor, M.E.: Near peak scattering and the corrected Kirchhoff approximation for a convex obstacle. Adv. Math. 55(3), 242-315 (1985)

8. Melrose, R.B., Taylor, M.E.: The radiation pattern of diffracted wave near the shadow boundary. Commun. P.D.E. 11(6), 599-672 (1986) 
9. Melrose, R.B., Taylor, M.E.: Boundary problems for the wave equation with grazing and gliding rays. Preprint, 1987

10. Nussenzveig, H.M.: High frequency scattering by an inpenetrable sphere. Ann. Phys. 34, 23-95 (1965)

11. Zworski, M.: High frequency scattering by a convex obstable. Duke Math. J. 61(2), 545-634 (1990)

Communicated by S.-T. Yau 